\title{
An efficient statistical strategy to monitor a robot swarm
}

\author{
Fouzi Harrou, Member, IEEE, Belkacem Khaldi, Ying Sun, and Foudil Cherif
}

\begin{abstract}
Detecting anomalies in a robot swarm play a core role in keeping the desired performance, and meeting requirements and specifications. This letter deals with the problem of detecting anomalies in a robot swarm. In this regards, an unsupervised monitoring approach based on principal component analysis and k-nearest neighbor is proposed. The principal component analysis model is employed to generate residuals for anomaly detection. Then, the residuals are examined by computing the proposed exponentially smoothed k-nearest neighbor statistic for the purpose of anomaly detection. Here, instead of using parametric thresholds derived based on the Gaussian distribution, a nonparametric decision threshold is computed using the kernel density estimation method. This provides more flexibility to the proposed detector by relaxing assumption on the distribution underlying the data. Tests on data from ARGoS simulator show efficient performance of the proposed mechanism in monitoring a robot swarm.
\end{abstract}

\section{INTRODUCTION}

Swarm robotics has been raised as a multi-robotic subfield that seeks to mimic natural swarm behaviors through the use of relatively simple artificial/physical agents [1], [2]. It mainly addresses both the design and the control models of the agents in a manner that the overall collective behavior of the swarm emerges from the simple local interaction rules among agents as well as between the agents and their surroundings. In fact, swarm robotics systems are strongly operating without central controllers and are expected to share several swarm features such as scalability, robustness, and flexibility. In its early age, swarm robotics systems have been usually used to replicate biological swarming tasks [3], [4] including aggregation, foraging, and pattern formation. Nowadays, much research has been directed to address the real application of such robotics systems in our daily life. For instance, they can be beneficially involved in tasks like rescuing in emergency situations, collaborative search and exploration, agricultural foraging, military missions, products delivery, and warehouse managing [1], [3], [5].

It has been early argued that using swarm robotics systems in performing such tasks are highly robust, in the way that the performance of the overall collective behavior of the swarm will not be entirely affected by a complete failure of individual robots. However, new studies suggested that swarm robotics systems are susceptible to be less tolerance to specific faults

F. Harrou and Y. Sun are with King Abdullah University of Science and Technology (KAUST) Computer, Electrical and Mathematical Sciences and Engineering (CEMSE) Division, Thuwal, 23955-6900, Saudi Arabia e-mail: fouzi.harrou@kaust.edu.sa

B. Khaldi and F. Cherif are with LESIA Laboratory, Department of Computer Science, University of Mohamed Khider, R.P. 07000 Biskra, Algeria called partial failures [6], [7]. In more details, a failing of one robot' sup-component such motors, for example, is able to have a serious effect on the performance of the entire motion of the swarm while compared to other failures modes (e.g.: a robot' IR sensor fault mode and a robot' power fault mode). Thus a critical consideration of the consequence leading to partial robot failures should be taken into account in the analysis of fault tolerance in robot swarm, and in the design of robot control robots swarm [7].

Technically, a fault is any unpredicted change that occurs in system function and which disrupts normal operation, resulting in unacceptable performance degradation [8]. Therefore, the binary decision process that approves whether an error has occurred in a system is known as fault detection. Indeed, faults in robotic swarm systems are generally hard to avoid due to the scalability and robustness of these systems [1]-[3]. This results in raising a new challenge in the literature of swarm robotics. As consequence, increasing attention for robots swarm monitoring is leading to the development of many fault detection techniques, which belong to either endogenous or exogenous fault detection classes [9], [10].

Detecting faults in a swarm robot play a core role to achieve acceptable performances and meet with the desired requirements and specifications. In endogenous fault detection approaches, usually, a local decision is made by each robot if one of its own sub-components fails. In this context, Skoundrianos et al. [11] applied a local neural network model to detect failures in the wheels' motor of a mobile robot. Canham et al. [12] were able to model an immune-based fault detection technique to identify errors occurring in two kinds of mobile robots. Christensen et al. [13], [14] designed a fault-detection method based on a time-delay neural network technique in a small mobile robot called s-bot. Mokhtar et al. [15] took inspiration from the functioning of dendritic immune system cells and proposed an error detection approach applied to robotic swarm systems. Generally, endogenous fault detection approaches ignore the interaction among robots, which is not the case in major tasks performed by swarm robotics systems. This typically led to a misleading diagnosis that may result in such techniques. As an example, failures such as a software bug or a dead battery can occur in a robot that cannot detect it alone. Moreover, even if a robot is ready to detect this type of failure, it may not be well broadcasted to the other robot of the swarm in case of malfunction of the communication device of the robot.

An alternative philosophy to mitigate these drawbacks was the introduction of exogenous fault detection methods. These 
methods used information available about the neighborhood of the swarm to collectively decide whether a robot among the swarm is faulty [16]. Therefore, they allow one or more robots of the swarm to detect failures occurring in another robot. Many works on the basis of these approaches have been suggested. For example, an exogenous fault detection model inspired by firefly behavior has been proposed by Christensen et al. [16] to detect failures on real robot swarms. On the basis of Artificial Immune System (AIS) techniques, Jakimovski et al. [17] suggested a method inspired by the intracellular signaling network and the T-Cell Receptor mechanisms to detect abnormalities in a system of autonomous robots swarm. Whereas Tarapore et al. [18] assessed the performance of an AIS based exogenous fault detection model on a completely different swarm robotics case study behaviors such as aggregating, dispersing, harming, and flocking. In other studies, Khadidos et al. [19] reported an exogenous fault detection approach based on a model that communicates sensor readings and robot motor speeds to their neighbors. Millard et al. [20] were able to detect run-time faults on a swarm robot system by comparing a local prediction model implemented in each robot with the actual behavior of other robots of the swarm.

In general, endogenous and exogenous anomaly detection methods can be designed using mathematical models or datadriven implicit models. In model-based schemes, usually an analytical model of the expected behavior of the system is first established, and then an anomaly is detected by comparing the model prediction with the actual behavior of the system [21]. Unfortunately, to implement such accurate models on robotic swarm systems are hard to design and can be timeconsuming [22]. Therefore, implicit data-based models are a good alternative, especially in the absence of explicit models, and in cases where the only available resource for process monitoring are measurement signals [23]. Mainly, the idea behind these methods is to construct an empirical model using the available process measurements and use it to efficiently extract relevant features in the design of monitoring schemes. Among the well-known methods that form the basis of datadriven models are computational intelligence and machine learning techniques. One of those techniques is multivariate statistical process control (MSPC) charts, which have been commonly used in process industries [24].

With an objective to enhance profitability, efficiency, and reliability of a robot swarm, this paper presents principle component analysis (PCA) in combination with kNN and exponential smoothing for anomaly detection. While PCA have been widely used to detect faults in various process industries, so far little work has been done with such charts to monitor robotic swarm systems [24]. In other words, the aim of this study is merging the advantages of univariate monitoring schemes, PCA, and kNN to improve their performances and broaden their practical applicability. In this approach, faulty robots are detected using PCA that generates residuals, and kNN-based methods are employed to evaluate uncorrelated residuals for anomaly detection. Two new charts, kNN-ES and Shewhart methods, are proposed as improved alternatives for fault detection. The basic idea is to apply the univariate monitoring chart (exponential smoothing and 3-sigma rule) to evaluate the $\mathrm{kNN}$ distances for anomaly detection. As the application of the univariate methods, such as exponential smoothing and Shewhart, require uncorrelated and Gaussian residuals to get a consistent result. The superior performance of the new combined fault detection mechanisms is demonstrated via the ARGoS simulator. Results suggest that the detection performance can be effectively enhanced by using nonparametric threshold instead of the use of decision thresholds based on the Gaussian assumption. It has been shown that the proposed approach is able to efficiently detect a different kind of errors including abrupt errors, drift errors, random walks and complete stop errors.

The rest of this letter is arranged as follows. The designed kNN-based procedures are described in Section II. Section III presents the experimental results. Finally, conclusions are provided in Section IV.

\section{THE $k$ NN-BASED MONITORING MECHANISMS}

\section{A. Features extraction with PCA}

Principal Component Analysis (PCA), a dimensionality reduction approach, is becoming an increasingly popular modeling framework to learn relevant and crucial features from multivariate data. By projecting the process variables into lower-dimensional subspace, PCA enables revealing the crosscorrelation inherent among process variables [?]. Let consider $\mathbf{X}=\left[\mathbf{x}_{1}^{T}, \ldots, \mathbf{x}_{n}^{T}\right]^{T} \in R^{n \times m}$ be a scaled data gathered from a swarm robot having $n$ observations and $m$ variables. Based on the PCA model, the data matrix $\mathbf{X}$ can be expressed as a sum of the approximated matrix, $\widehat{\mathbf{X}}$, and residual data, $\mathbf{E}$.

$$
\mathbf{X}=\mathbf{T W}^{T}=\sum_{i=1}^{k} t_{i} w_{i}^{T}+\sum_{i=k+1}^{m} t_{i} w_{i}^{T}=\widehat{\mathbf{X}}+\mathbf{E}
$$

where $\mathbf{T} \in R^{n \times m}$ represent a matrix of the principal components (PCs) and $\mathbf{W} \in R^{m \times m}$ is the loading matrix. In the presence of cross-correlated multivariate data, $\mathbf{X}$, as the case data from a swarm robot, the first ' $k$ ' PCs (where $k<m$ ) are sufficient for preserving relevant information in the original data. One important step in PCA model development is to select the number of PCs. For this purpose, the cumulative percentage variance $(\mathrm{CPV})$ procedure is utilized due to its simplicity and accuracy.

The loading matrix is frequently calculated through Singular Value Decomposition of the covariance matrix $\mathbf{S}$ of the data $\mathrm{X}$ :

$$
\mathbf{S}=\frac{1}{n-1} \mathbf{X}^{T} \mathbf{X}=W \Lambda W^{T} \text { with } W W^{T}=W^{T} W=I_{n} .
$$

where, $\Lambda=\operatorname{diag}\left(\sigma_{1}^{2}, \ldots, \sigma_{m}^{2}\right)$ is a matrix comprising eigenvalues of $\mathbf{S}$ arranged diagonally in decreasing order. The eigenvalues $\lambda_{i}$ are equal to the variance of the PC $t_{i}, \sigma_{i}^{2}$.

Th residual matrix play a core role to uncover abnormal features in the inspected system. For the purpose of anomaly detection, the generated residuals based on the developed PCA reference model will be evaluated by the proposed $\mathrm{kNN}$-based mechanisms. 


\section{B. Anomaly detection with $k N N$-based mechanisms}

The $k \mathrm{NN}$ is a well-known and yet simple technique that is more appropriate for use in classification problems studies where the data collected is not linearly separable or has a nonGaussian distribution [25]. In general, the technique looks for the similarity between a learning set and its corresponding observation set. It specifically seeks for assigning a label to a new sample basing on the $\mathrm{k}$ training samples that are next to that new sample, according to a specific distance measure [25], [26]. When applied to detect faults in industrial processes, $\mathrm{He}$ and Wang [27] proposed the first $k \mathrm{NN}$-based fault detection $(k \mathrm{NN}-\mathrm{FD})$ approach by only using the available normal data set rather than the fault data set. This due to the fact that in reality, it is impracticable to collect fault data training set with limitless labeled fault data. The idea behind the He and Wang' $k$ NN-FD approach is how to define a threshold with a certain level of confidence and to use this threshold as a criterion for labeling the new sample as a fault if the distance to its $k$ closest neighbors is greater than the threshold. Otherwise, the new sample is considered as normal [28].

In the proposed PCA-based kNN mechanisms, the PCA model has implemented to model multivariate processes. Based on fault-free measurements, the reference PCA model is designed and then adopted for fault detection. Indeed, after getting the reference PCA model, it is applied to new testing data to get residuals for fault detection. For new testing measurements, $\mathbf{X}=\left[x_{1}, \ldots, x_{t}, \ldots, x_{n}\right]$, the residual , $\mathbf{E}=\left[e_{1}, \ldots, e_{t}, \ldots, e_{n}\right]$, are generated via PCA model as,

$$
\mathbf{E}=\mathbf{X}-\hat{\mathbf{X}},
$$

where $\hat{\mathbf{X}}=\left[\hat{x}_{1}, \ldots, \hat{x}_{t}, \ldots, \hat{x}_{n}\right]$ are the PCA predicted values. The kNN-based detector is applied to residuals used to indicate an unusual shift from the reference PCA model. Specifically, the kNN approach is applied to residuals and construct the decision statistic for anomaly detection as described next. The block diagram of the designed anomaly detection mechanism is schematized in Figure 1. It is composed of two main parts, each having three steps as follow:

- (i) Model building phase

1) For every input data, the residuals e are obtained by the reference PCA model.

2) For each query, $X_{i}$, in the trainig data set, identify its $k$ NNs according to a specific distance metric $d_{i j}$ (e.g. Eucludian and Manhattan).

3) For that $X_{i}$ compute the average squared distance, $D_{i}^{2}$, to its KNNs as follow [29]:

$$
D_{i}^{2}=\frac{1}{k} \sum_{j=1}^{k} d_{i j}^{2} .
$$

Where $d_{i j}^{2}$ is the squared distance from the sample, $X_{i}$, to its $\mathrm{j}^{\text {th }}$ closest neighbor.

4) For the same query, $X_{i}$, define the threshold, $D_{\alpha}^{2}$. When the distribution of $k \mathrm{NN}$ distances in the data training set is assumed to be normally distributed, $D_{\alpha}^{2}$ can be defined using the three-sigma control chart: $D_{\alpha}^{2}=\mu_{D}+$ $3 \sigma_{D}$, where $\mu_{D}$ and $\sigma_{D}$ represent the mean and standard

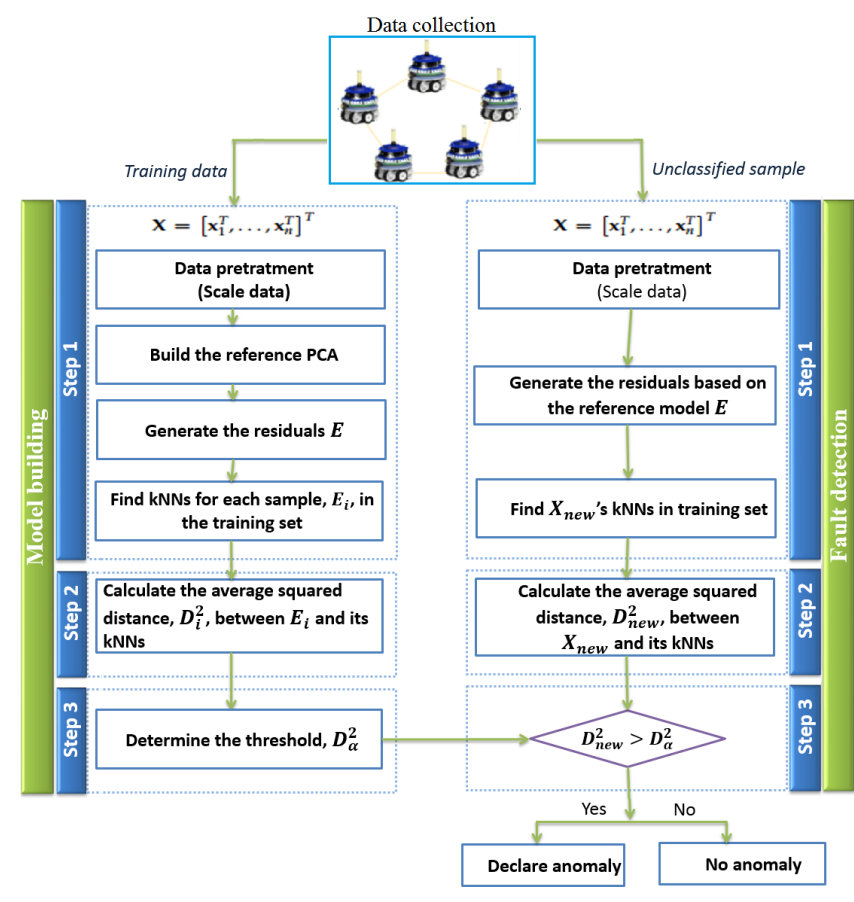

Fig. 1: A block diagram of the proposed $k \mathrm{NN}$-based monitoring mechanism.

deviation of the distances of $k \mathrm{NN}$ [29]. Otherwise, the distribution of $k \mathrm{NN}$ distances can be estimated using a Kernel Density Estimation (KDE) technique, where its $(1-\alpha)^{t h}$ quartile can be chosen to define $D_{\alpha}^{2}$.

- (ii) Fault detection phase

1) For a new unclassified query, $X_{n e w}$, find its $k \mathrm{NNs}$ in the training data set using the same distance metric used in the $1^{\text {st }}$ step of the model building part.

2) using Eq.4, compute the squard distance $D_{\text {new }}^{2}$.

3) Compare $D_{n e w}^{2}$ with the control limit $D_{\alpha}^{2}$. Anomaly is declared if $D_{\text {new }}^{2}$ exceeds $D_{\alpha}^{2}$. Otherwise, normality is considered.

1) $k N N$-Shewhart approach: We used $k \mathrm{NN}$ to compute the deviation between the current residual observation and residuals of fault-free measurements. Generally speaking, in absence of faults, $k \mathrm{NN}$ distances fluctuate around zero, while $k \mathrm{NN}$ distances diverge from zero in the presence of faults. Let's define the vector of $k \mathrm{NN}$ distances as computed: $D=$ $\left[d_{1}, \ldots, d_{j}, \ldots, d_{n}\right]$. Two monitoring charts are proposed in this studied to compute the decision threshold based on which faults can be detected. In the first approach, kNN-Shewhart, the kNN distances are evaluated using Shewhart chart to detect faults. It is performed by calculating the decision threshold using the three sigma rule.

$$
H=\mu_{D}+3 \sigma_{D}
$$

where $\mu_{D}$ and $\sigma_{D}$ are the mean and standard deviation of $k \mathrm{NN}$ distances using free-flow training measurements, and normality is assumed implicitly in this three-sigma methodology.

2) kNN-ES approach: The second approach is designed by applying the exponential smoothing (ES) chart to kNN 
distances. Indeed, $\mathrm{kNN}$ distances between the actual ANFISPLS residual observation and the normal training residual samples reflect inconsistencies between the healthy and faulty operating conditions. Then, the ES chart is used to evaluate $\mathrm{kNN}$ distances for fault detection. The $k \mathrm{NN}-\mathrm{ES}$ charting statistic is defined as follows:

$$
z_{d_{t}}=\nu d_{t}+(1-\nu) z_{d_{t-1}}
$$

where $z_{d_{0}}=\mu_{0}^{D}$ is the anomaly-free mean of the vector of $k \mathrm{NN}$ distances. $\nu(0<\nu 1)$ is the weighting factor. The value of $\nu$ is usually selected in the interval $0.1 \leq \nu \leq 0.3$ to detect small changes in practice [30]. The $k$ NN-ES approach flags faults when the $k \mathrm{NN}-\mathrm{ES}$ statistic given in (6), $z_{t}^{k N N}$, is exceed the control limits, $h$, defined as

$$
h=\mu_{0}^{k N N}+\kappa \sigma_{0}^{k N N} \sqrt{\left(\frac{\nu}{(2-\nu)}\left[1-(1-\nu)^{2 t}\right]\right.},
$$

where $\kappa$ is a multiplier of standard deviation of $k \mathrm{NN}$ distances, $\sigma^{k N N}=\sigma_{0}^{k N N} \sqrt{\left(\frac{\nu}{(2-\nu)}\left[1-(1-\nu)^{2 t}\right]\right.}$. Commonly, the selection of small values of $\nu$ is appropriate to increase the sensitivity to small deviation in $k \mathrm{NN}$ distances, while the use of large values of $\nu$ is appropriate for detecting larger changes in the $k \mathrm{NN}$ distances.

The conventional parametric Shewhart and ES control procedures are suitable only when the normality assumption is valid. Indeed, the $k \mathrm{NN}$ algorithm is an efficient technique to identify the similarity between normal and abnormal features. However, associating it with a decision threshold based on the Gaussian distribution could degrade its performance. To mitigate this limitation, the kernel density estimation [31] was used to estimate the distribution of $k \mathrm{NN}$-based distances and compute a nonparametric threshold for traffic monitoring. Specifically, from the distribution of the decision statistic, $z_{d_{t}}$, a non- parametric threshold of the kNN-ES mechanism is defined as the $(1-\alpha)$-th quantile of the estimated distribution of kNN-ES statistic obtained by kernel density estimation (KDE). The nonparametric threshold is computed using anomaly-free data.

\section{Simulation RESUlts}

\section{A. Application to swarm robotics formation control problem-} atic

To test the effectiveness of the designed approach, data from 6 foot-bots robots performing an artificial physical based circle formation controller are collected [32] using the ARGoS simulator [33]. A foot-bot robot (See Fig.2) is a 2-wheels differential drive robotic platform that was designed within the context of the Swarmanoid project ${ }^{1}$ for conducting swarm robotics research studies [34]. It is about $14 \mathrm{~cm}$ and $29 \mathrm{~cm}$ of dimension. The foot-bot comes with a main processor board that is based on a $533 \mathrm{MHz}$ i.MX31 ARM 11 processor family. The board is of $64 \mathrm{MB}$ of Flash,128 MB of RAM, and 2.0 USB host controller [35]. The foot-bot comes essentially with 12 RGB LEDs for displaying coloured patterns to other robots, an omni-directional camera for perceiving displayed coloured

${ }^{1}$ www.e-swarm.org patterns, 24 IR sensors for detecting obstacles and proximities, and a range-and-bearing ( $\mathrm{RAB}$ ) communication device for inter-robots message exchanging. The sensors/actuators used to implement the circle formation controller are mainly the RAB communication device and the wheels encoders. The RAB device is specifically used to detect neighbors and compute the total artificial physical force exerted on a robot by its sensed neighbors.

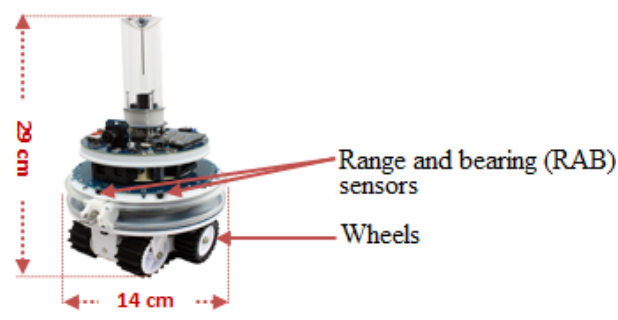

Fig. 2: The real foot-bot robot with the used sensors/actuators.

The resulting artificial force will then serve as input to actuate the robot's wheels through calculating the forward speed of the left and right wheels. A flowchart schema of the artificial physical based circle formation controller implemented on each foot-bot robot is illustrated in Fig.3. Details on the values of the controller parameters can be found in [24], [32].

The ARGoS simulation results within the 6 foot-bots robots were conducted for a total duration of 1500 time steps (150 seconds). The robots were placed randomly inside a rectangular bounded area and their headings are set to be arbitrary. Robots trajectories for one particular simulation of performing a circle formation task are illustrated in Fig.4. During the ARGoS simulation, readings from inputs/outputs of the circle formation controller, and which are strongly related to the RAB device and the wheels' encoders of each foot-bot robot, are collected in Table I. These readings will be further used as training data for the proposed fault detection monitoring approach.

TABLE I: Readings collected during performing a circle formation task using the ARGoS Simulation

\begin{tabular}{ll}
\hline Reading & Description \\
\hline$v_{r_{i}}$ & Right Wheel Forward Speed \\
$v_{l_{i}}$ & Left Wheel Forward Speed \\
$F_{i}^{v v c}$ & Virtual Viscoelastic Force length \\
$\angle F_{i}^{v v c}$ & Virtual Viscoelastic angle \\
\hline
\end{tabular}

To construct the PCA model 1500 samples are gathered from a robot swarm of 6 robots running under nominal conditions. From each robot, viscoelastic force-length, and viscoelastic force angle are collected. The CPV method is used to determine the optimum number of PCs in the PCA model. Three PCs capturing $96 \%$ of the total variability in the inspected swarm, are chosen for developing the PCA model. The designed PCA model that reflects the healthy behavior of the swarm robot will be used for fault detection purposes. Four additive anomalies are considered in this study to test the 


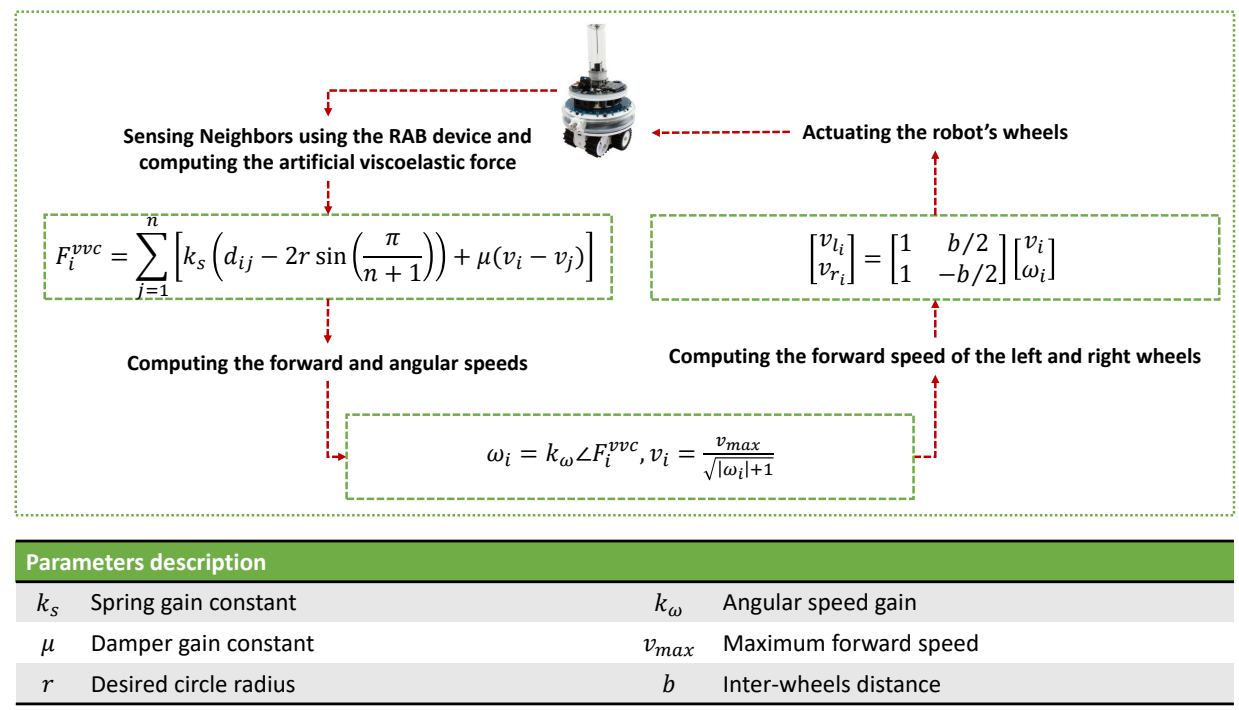

Fig. 3: A flow chart of the artificial viscoelastic based circle formation controller.

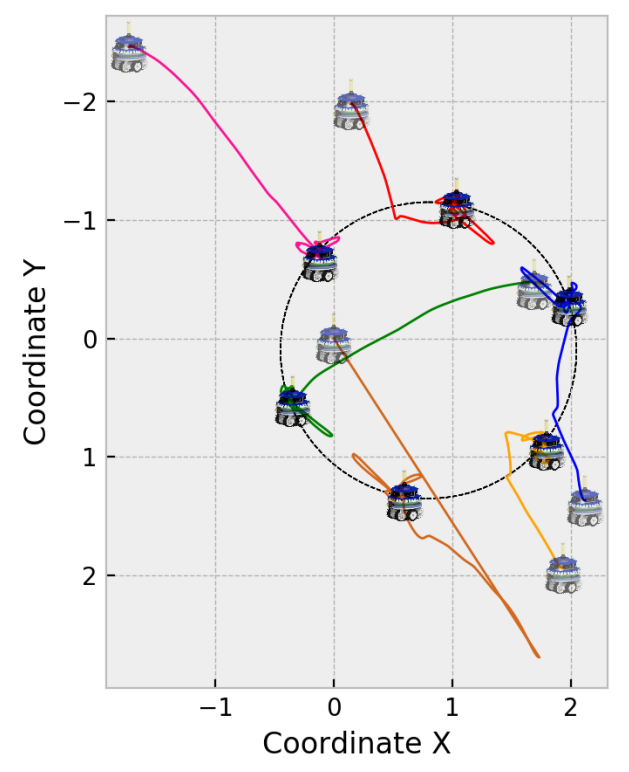

Fig. 4: Plots of robots trajectories during performing the artificial viscoelastic based circle formation controller.

performance of the proposed schemes: sensor bias, drift fault, intermittent fault, random walk, and complete stop faults.

To quantify the detection quality of our proposed fault detection approach, we rely on a set of well-known fault detection performance metrics that are commonly used in the context of binary detection problems. In such type of decision, a $2 \times 2$ confusion matrix, reporting the number of true positives (TP), false positives (FP), false negatives (FN), and true negatives (TN) is by convention used to summarize a detector's performance [36]. As consequence, many common performance metrics are formed on the basis of this matrix. To assess quantitatively the detection efficiency of the proposed procedures, the following metrics will be used: true positive rate (TPR), false positive rate (FPR), accuracy, and area under the curve (AUC). Figure 5 illustrates a confusion matrix and summarizes equations of the main related metrics that are commonly used to assess the quality of a binary decision method [36] and which will be used to assess the performance of our PCA-kNN based fault detection approach.

\section{B. Faults Generation}

This subsection explains how we inject faulty data in the sensor/encoder sub-components of one or more footbots during performing a circle formation task. Formally, any measurement, $m(t)$, of a given system is commonly defined as $m(t)=r(t)+f(t)$, where $r(t)$ is the true or the real value of the measurement and $f(t)$ is a value of a systematic error at time $t$ caused mainly by a malfunction in the corresponding sub-component. This systematic error can be generated using different fault modes. To this end, the faults types described in the sub-sections below are used to inject faulty measurements to the real measures of the motor encoders and the RAB sensors. The faulty data of each type of fault is injected in real time in ARGoS during performing the desired circle formation task.

1) Abrupt fault type: This fault mode commonly occurs in practice. The measurement in this mode can be formally defined in time as:

$$
m(t)= \begin{cases}r(t), & t<t_{s} \\ r(t)+c, & t \geq t_{s}\end{cases}
$$

where $c$ is a constant deviation that occurs from the starting time $t_{s}$. 


\begin{tabular}{|c|c|c|c|c|c|}
\hline \multicolumn{4}{|c|}{ The confusion matrix } & \multicolumn{2}{|c|}{ Performance Metrics } \\
\hline & & & & & \\
\hline$\pi$ & Positive & Negative & Row Totals & $T P R=\frac{t p}{R P}$ & $F P R=\frac{f p}{R N}$ \\
\hline 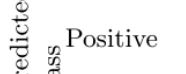 & $\begin{array}{c}\text { true positive } \\
(t p)\end{array}$ & $\begin{array}{c}\text { false positive } \\
(f p)\end{array}$ & $P P=t p+f p$ & Accuracy $=\frac{t p+t n}{R P+R N}$ & Precision $=\frac{t p}{P P}$ \\
\hline م $\frac{\pi}{\mathrm{U}}$ Negative & $\begin{array}{c}\text { false negative } \\
\quad(f n)\end{array}$ & $\begin{array}{l}\text { true negative } \\
(t n)\end{array}$ & $P N=f n+t n$ & $F_{1} S$ core $=2 * \frac{\text { Precision } * T P R}{\text { Precision }+T P R}$ & $A U C=\frac{T P R-F P R+1}{2}$ \\
\hline
\end{tabular}

Fig. 5: Confusion matrix and associated commonly used performance metrics.

2) Drift fault type: This failure mode refers to the magnitude of fault that changes linearly in time. Here, the measurement is modeled as follow:

$$
m(t)= \begin{cases}r(t), & t<t_{s} \\ r(t)+\lambda\left(t-t_{s}\right), & t \geq t_{s}\end{cases}
$$

where $\lambda$ is a constant and $t_{s}$ is the fault starting time.

3) Stop Fault type: In this type of failure, the subcomponent responsible to get a measurement will completely dysfunctioning. It can be mainly modeled as zeroing the true measure starting from the fault starting time $t_{s}$ as follow:

$$
m(t)= \begin{cases}r(t), & t<t_{s} \\ 0, & t \geq t_{s}\end{cases}
$$

4) Random walk Fault type: This refers to a random movement affecting the motors encoders of the inspected robot. It can be simulated by adding, from the starting fault time $t_{s}$, random Gaussian noise of the form $\mathcal{N}(0, \sigma)$ to the real measurement as follow:

$$
m(t)= \begin{cases}r(t), & t<t_{s} \\ r(t)+\mathcal{N}(0, \sigma), & t \geq t_{s}\end{cases}
$$

\section{Faults Detection Results}

To test the detection capacity of the proposed techniques, 300 samples per each fault type case study are gathered from the monitored swarm robotics system. Faulty data are injected in real time at different intervals of the total ARGoS simulation time as discusses in subsection.III-B. All the results are ploted as radar charts of six axes representing the commonly used performance metrics illustrated in Fig.5.

1) Abrupt faults: In this scenario, it is considered that the viscoelastic force-length of the first robot, $x_{1}$, is abruptly failed from observation 200 to 300 . To simulate abrupt fault, a small bias of $10 \%$ of the total variation in the raw measurements is incorporated in the raw measurements, $x_{1}$. A snapshot from the ARGoS simulator emulating this case study is illustrated in Fig.6a. Detection results of kNN-based techniques are shown in Table II and Fig.7a. As the occurred fault is relatively large, the four schemes provide good detection with an AUC greater than 0.96 . The results indicate that the $\mathrm{kNN}$-based ES and Shewhart with the nonparametric thresholds (See red and black lines in the figure) provide slightly improved detection results compared to the corresponding Gaussian distributionbased thresholds.

The testing data of the second example is contaminated with a bias of $5 \%$ of the total variation in the raw measurements from sample ranges 200 to 300 . The corresponding ARGoS-

\begin{tabular}{|c|c|c|c|c|c|c|}
\hline Approach & TPR & FPR & Accuracy & Precision & F1Score & AUC \\
\hline$k \mathrm{NN}-\mathrm{EWMA}{ }^{n p}$ & 0,980 & 0,016 & 0,983 & 0,926 & 0,952 & 0,982 \\
\hline$k$ NN-EWMA ${ }^{p a}$ & 0,980 & 0,056 & 0,95 & 0,781 & 0,870 & 0,962 \\
\hline$k \mathrm{NN}-$ Shewhart ${ }^{n p}$ & 0,980 & 0 & 0,997 & 1 & 0,990 & 0,990 \\
\hline$k \mathrm{NN}-$ Shewhart ${ }^{p a}$ & 0,980 & 0,020 & 0,98 & 0,909 & 0,943 & 0,980 \\
\hline
\end{tabular}
based simulation snapshot for this case study id shown in
TABLE II: Abrupt faults detection results with bias $=10 \%$

Fig.6b. Table II and Fig.7b displays the detection results of KNN-based Shewhart and EWMA mechanisms (with parametric and nonparametric thresholds). The results highlight that the detection capability is improved by using the nonparametric k-NN-based approaches.

TABLE III: Abrupt faults detection results with bais $=5 \%$

\begin{tabular}{lllllll}
\hline Approach $^{n}$ & TPR & FPR & Accuracy & Precision & F1Score & AUC \\
\hline NNN-EWMA $^{n p}$ & 0,980 & 0,012 & 0,987 & 0,943 & 0,962 & 0,984 \\
$k_{\text {NN-EWMA }}{ }^{n a}$ & 0,980 & 0,052 & 0,953 & 0,794 & 0,877 & 0,964 \\
$k_{\text {NN-Shewhart }}^{n p}$ & 0,922 & 0 & 0,987 & 1 & 0,959 & 0,961 \\
$k_{\text {NN-Shewhart }}{ }^{p a}$ & 0,941 & 0,020 & 0,973 & 0,906 & 0,923 & 0,961 \\
\hline
\end{tabular}

In the third scenario, a 3\% bias fault is introduced in sensor $x_{1}$ (See Fig.6c for an ARGoS-based simulation snapshot). Detection results of the four methods are summarized in Table IV and Fig 7c. The corresponding results confirm also the outperformance of the nonparametric kNN-ES mechanism compared to the other charts. This highlights the benefit of exponentially smoothing $\mathrm{kNN}$, which permit including all available information from past and actual data in the decision process. Also, from this case study, it can be concluded that kNN-based ES and Shewhart chart with nonparametric thresholds computed via KDE provide improved detection performance compared to their parametric thresholds-based counterparts. Therefore, using PCA-KNN schemes with nonparametric decision thresholds for anomaly detection are more efficient compared to using parametric thresholds determined based on the Gaussian assumption. The lowest prediction performance was obtained for the kNN-based Shewhart schemes (parametric and nonparametric) since they are based only on the actual residuals for the decision and ignore the past

\begin{tabular}{|c|c|c|c|c|c|c|}
\hline Approach & TPR & FPR & Accuracy & Precision & F1Score & AUC \\
\hline$k$ NN-EWMA ${ }^{n p}$ & 0,902 & 0,020 & 0,967 & 0,902 & 0,902 & 0,941 \\
\hline$k$ NN-EWMA ${ }^{p a}$ & 0,941 & 0,088 & 0,917 & 0,686 & 0,793 & 0,926 \\
\hline$k \mathrm{NN}-$ Shewhart ${ }^{n p}$ & 0,667 & 0,000 & 0,943 & 1 & 0,8 & 0,833 \\
\hline$k \mathrm{NN}-$ Shewhart ${ }^{p a}$ & 0,765 & 0,020 & 0,943 & 0,886 & 0,821 & 0,872 \\
\hline
\end{tabular}
information.

TABLE IV: Abrupt faults detection results with bias $=3 \%$

2) Intermittent Faults: The aim of this case is to test the detection capability of the PCA-based kNN schemes when intermittent faults happened. To do so, intermittent anomalies 


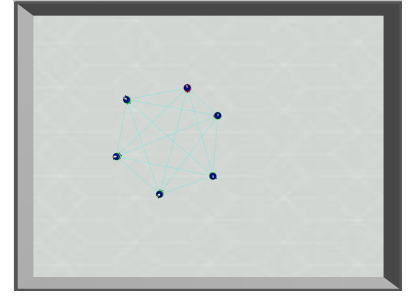

(a) With bais $=10 \%$

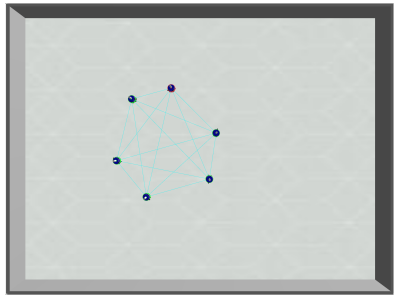

(b) With bais $=5 \%$

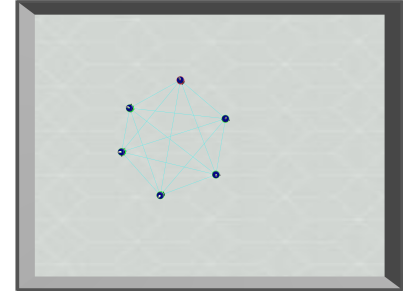

(c) With bais $=3 \%$

Fig. 6: ARGoS-based simulation snapshots at a sample $\in[200,300]$ with an abruptly faulty robot (in red colour).

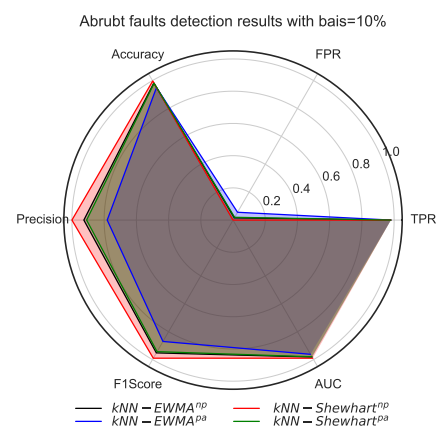

(a)

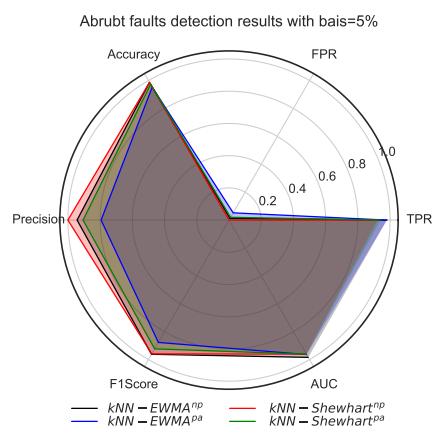

(b)

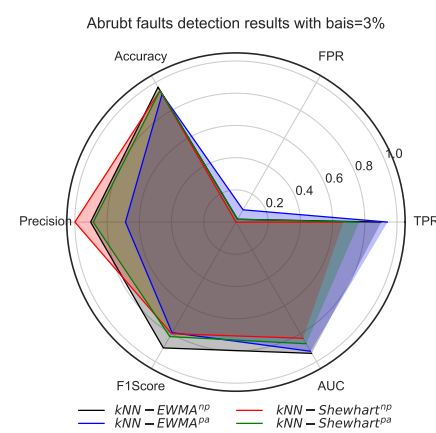

(c)

Fig. 7: Abrupt faults detection results.

with amplitude $5 \%$ of the total variation of raw measurements are added to the testing measurements for samples 50 to 100 , and bias of $10 \%$ for samples 150 to 200 . Figure 8 displays snapshots from the ARGoS simulator for these case studies correspondingly. Results of the four mechanisms are given in Table V and Fig.9. All detectors provide satisfactory results with an AUC greater than $\mathrm{AUC}=0.93$.

TABLE V: Intermittent faults detection results with $5 \%$ and $20 \%$

\begin{tabular}{|c|c|c|c|c|c|c|}
\hline Approach & TPR & FPR & Accuracy & Precision & F1Score & AUC \\
\hline$k$ NN-EWMA ${ }^{n p}$ & 0,980 & 0,035 & 0,970 & 0,935 & 0,957 & 0,973 \\
\hline$k$ NN-EWMA ${ }^{p a}$ & 0,980 & 0,121 & 0,913 & 0,806 & 0,885 & 0,930 \\
\hline$k \mathrm{NN}-\mathrm{Sh}$ whart ${ }^{n p}$ & 1,000 & 0,020 & 0,987 & 0,962 & 0,981 & 0,990 \\
\hline$k \mathrm{NN}-\mathrm{Shewhart}{ }^{p a}$ & 1,000 & 0,040 & 0,973 & 0,927 & 0,962 & 0,980 \\
\hline
\end{tabular}

3) Gradual Faults: Here, the aim is to verify the potential of the designed schemes in sensing sensor drift faults. In this regard, early detection of drift faults before they propagate and seriously degrade the process is essential. For the experiment, a $1 \%$ drift fault is injected in $x_{1}$ at observation 150. An ARGoSbased simulation snapshot for this scenario is shown in Fig.10. The highest detection accuracies are achieved by the kNNbased schemes with nonparametric thresholds (Table VI and Fig 11). When anomalies are accurately sensed in time, this will be helpful to determine the root cause of the detected anomaly and make the necessary actions before a serious problem occurs in a robot swarm.

4) Random Walk: In this case, the efficacy of proposed kNN-based anomaly detectors is tested when a random walk fault happened in the supervised robot swarm. To do so, a random Gaussian noise with a variance of $\sigma=0.5$ is incorporated to the viscoelastic force length of the first robot,
TABLE VI: Gradual faults detection results

\begin{tabular}{|c|c|c|c|c|c|c|}
\hline Approach & TPR & FPR & Accuracy & Precision & F1Score & AUC \\
\hline$k$ NN-EWMA ${ }^{n p}$ & 0,973 & 0,000 & 0,987 & 1,000 & 0,986 & 0,987 \\
\hline$k$ NN-EWMA ${ }^{p a}$ & 0,973 & 0,080 & 0,947 & 0,924 & 0,948 & 0,947 \\
\hline$k \mathrm{NN}-S h e w h \operatorname{art}^{n p}$ & 0,980 & 0,000 & 0,990 & 1,000 & 0,990 & 0,990 \\
\hline$k \mathrm{NN}-$ Shewhart ${ }^{p a}$ & 0,980 & 0,027 & 0,977 & 0,974 & 0,977 & 0,977 \\
\hline
\end{tabular}

$x_{1}$, after observation 200 in the testing data (See Fig.12 for an ARGoS-based simulation scenario). Results of the four monitoring mechanisms are presented in Table VII and Fig 13. The proposed kNN-ES mechanism with nonparametric threshold successfully detect this fault wi for th the highest detection performance compared to the other detectors.

TABLE VII: Random Walk faults detection results

\begin{tabular}{|c|c|c|c|c|c|c|}
\hline Approach & TPR & FPR & Accuracy & Precision & F1Score & AUC \\
\hline$k$ NN-EWMA ${ }^{n p}$ & 1,000 & 0,000 & 1,000 & 1,000 & 1,000 & 1,000 \\
\hline$k$ NN-EWMA ${ }^{p a}$ & 1,000 & 0,075 & 0,950 & 0,870 & 0,930 & 0,963 \\
\hline$k \mathrm{NN}-$ Shewhart ${ }^{n p}$ & 0,940 & 0,005 & 0,977 & 0,989 & 0,964 & 0,968 \\
\hline$k \mathrm{NN}-\mathrm{Shewhart}{ }^{p a}$ & 0,960 & 0,025 & 0,970 & 0,950 & 0,955 & 0,968 \\
\hline
\end{tabular}

5) Complete Stop: This case focuses on testing the capability of the designed mechanisms in sensing a complete stop fault in a robot swarm. The complete fault happened in the case when a robot stops operating and disconnect totally from the swarm. In this scenario, a complete stop fault is considered. To this end, the viscoelastic force of the first robot is zeroed from observation 200. In Fig.14, a snapshot of this scenario simulated using the ARGoS simulator is shown. Table VIII and Fig.15 summarizes the performance of the four detectors. The results clearly show the benefitAt of exponentially smoothing kNN distances and using nonparametric threshold (Black and red color). From Table VIII and Fig 15, it can be see that the use of nonparametric decision threshold via KDE provides 


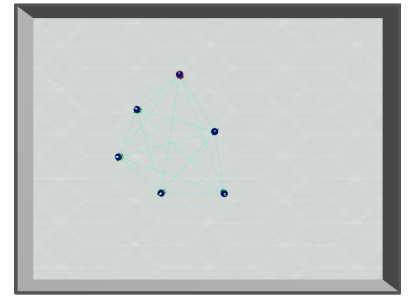

(a) With bias $=5 \%$ at a sample $\in[50,100]$.

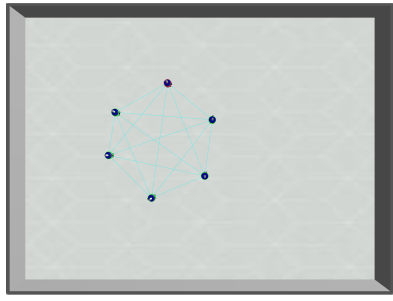

(b) With bias $=20 \%$ at a sample $\in[150,200]$

Fig. 8: ARGoS-based simulation snapshots with an intermittently faulty robot (in red colour).

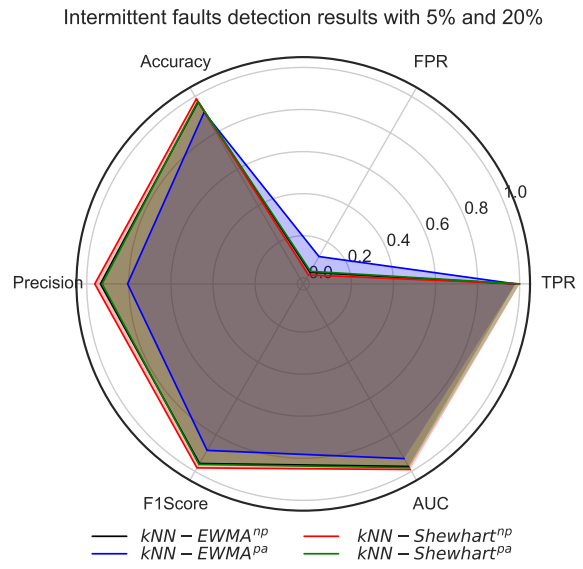

Fig. 9: Intermittent faults detection results.

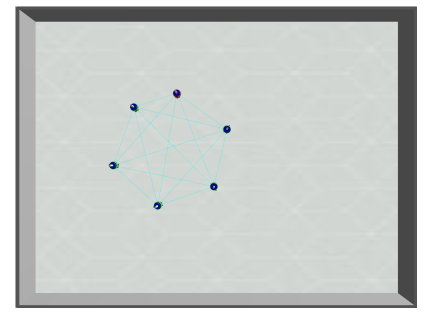

Fig. 10: ARGoS-based simulation snapshot at sample 150 with a gradually faulty robot (in red colour).

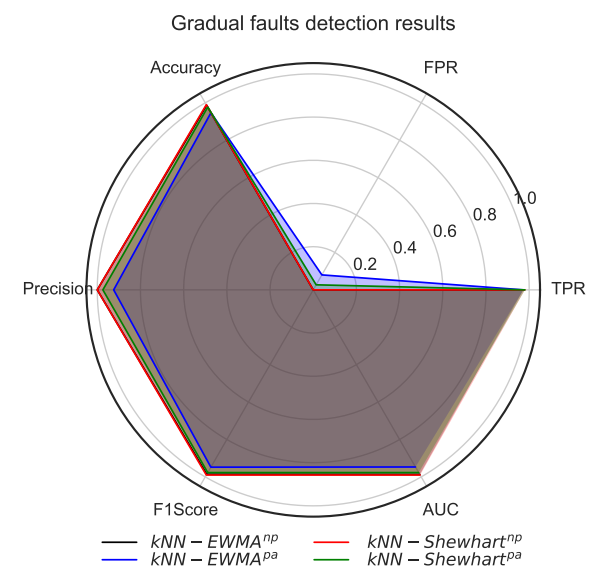

Fig. 11: Gradual faults detection results.

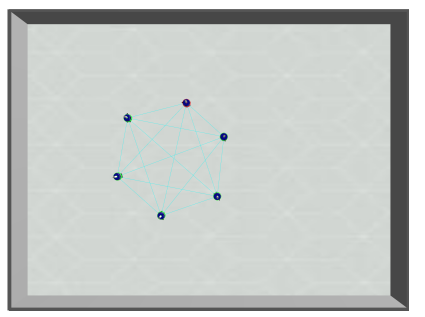

Fig. 12: ARGoS-based simulation snapshot at sample 200 with a randomly walking faulty robot (in red colour).

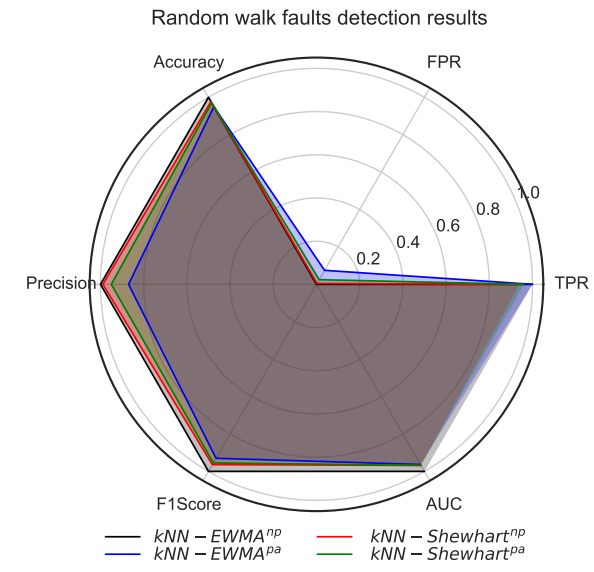

Fig. 13: Random walk faults detection results.

clear of complete stop fault. In this experiment kNN-ES detector provided a superior performance than $\mathrm{kNN}$-Shewhart detectors.

TABLE VIII: Complete Stop faults detection results

\begin{tabular}{|c|c|c|c|c|c|c|}
\hline Approach & TPR & FPR & Accuracy & Precision & F1Score & AUC \\
\hline$k$ NN-EWMA ${ }^{n p}$ & 1,000 & 0,000 & 1,000 & 1,000 & 1,000 & 1,000 \\
\hline$k$ NN-EWMA ${ }^{p a}$ & 1,000 & 0,075 & 0,950 & 0,870 & 0,930 & 0,963 \\
\hline$k$ NN-Shewhart ${ }^{n p}$ & 1,000 & 0,005 & 0,997 & 0,990 & 0,995 & 0,998 \\
\hline$k \mathrm{NN}-$ Shewhart ${ }^{p a}$ & 1,000 & 0,025 & 0,983 & 0,952 & 0,976 & 0,988 \\
\hline
\end{tabular}

\section{Conclusion}

Since anomalies and failures are inevitable in a robot swarm, they must be suitably detected and corrected. This paper proposed an unsupervised data-based mechanism based on PCA and kNN for fault detection in a robot swarm. Specifically, PCA is utilized to extract relevant features and generate residuals and $\mathrm{kNN}$-based schemes with their nonparametric 


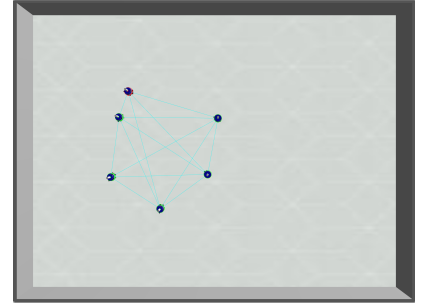

Fig. 14: ARGoS-based simulation snapshot at sample 200 with a completely stoping faulty robot (in red colour).

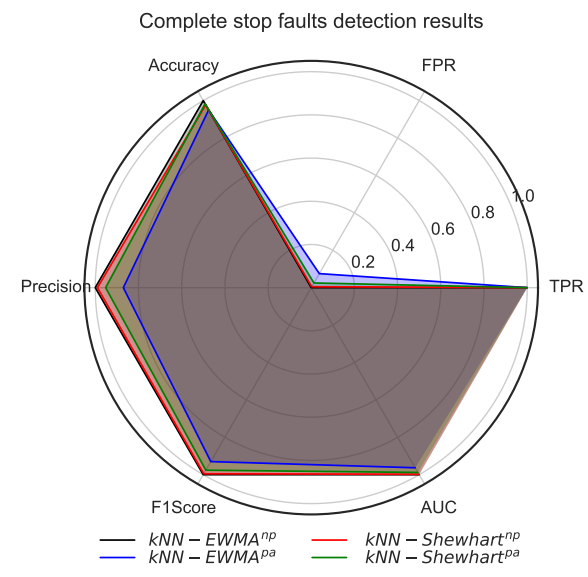

Fig. 15: Complete Stop faults detection results.

thresholds computed by $\mathrm{KDE}$ are applied to residuals for anomaly detection. The feasibility of the designed detection schemes is verified using data from ARGoS simulator. Results indicate the efficiency of the proposed schemes in detecting faults in a robot swarm. The results show that the detection results with nonparametric thresholds achieve better performance compared with the use of parametric thresholds based on the Gaussian distribution. Using KDE, the PDF underlying the decision statistic can be estimated more closely than assuming its Gaussianity. This allows building an efficient and flexible anomaly detector for monitoring a robot swarm.

\section{ACKNOWLEDGEMENT}

This publication is based upon work supported by the King Abdullah University of Science and Technology (KAUST) Office of Sponsored Research (OSR) under Award No: OSR2019-CRG7-3800.

\section{REFERENCES}

[1] Y. Tan and Z. Zheng, "Research advance in swarm robotics," Defence Technology, vol. 9, no. 1, pp. 18-39, 2013.

[2] M. Brambilla, E. Ferrante, M. Birattari, and M. Dorigo, "Swarm robotics: a review from the swarm engineering perspective," Swarm Intelligence, vol. 7, no. 1, pp. 1-41, 2013.

[3] B. Khaldi and F. Cherif, "An overview of swarm robotics: Swarm intelligence applied to multi-robotics," International Journal of Computer Applications, vol. 126, no. 2, 2015.

[4] B. Khaldi, F. Harrou, F. Cherif, and Y. Sun, "Self-organization in aggregating robot swarms: A dw-knn topological approach," Biosystems, vol. 165, pp. 106-121, 2018.
[5] _ - "Flexible and efficient topological approaches for a reliable robots swarm aggregation," IEEE Access, vol. 7, pp. 96372-96383, 2019.

[6] A. F. Winfield and J. Nembrini, "Safety in numbers: Fault tolerance in robot swarms," International Journal on Modelling Identification and Control, vol. 1, no. ARTICLE, 2006

[7] J. D. Bjerknes and A. F. Winfield, "On fault tolerance and scalability of swarm robotic systems," in Distributed autonomous robotic systems. Springer, 2013, pp. 431-444.

[8] R. Isermann, Fault-diagnosis systems: an introduction from fault detection to fault tolerance. Springer Science \& Business Media, 2006.

[9] A. Millard, J. Timmis, and A. Winfield, "Towards exogenous fault detection in swarm robotic systems," in Conference Towards Autonomous Robotic Systems. Springer, 2013, pp. 429-430.

[10] H. Lau, "Error detection in swarm robotics: A focus on adaptivity to dynamic environments," 2012.

[11] E. Skoundrianos and S. Tzafestas, "Finding fault-fault diagnosis on the wheels of a mobile robot using local model neural networks," IEEE Robotics \& Automation Magazine, vol. 11, no. 3, pp. 83-90, 2004.

[12] R. Canham, A. Jackson, and A. Tyrrell, "Robot error detection using an artificial immune system," in NASA/DoD Conference on Evolvable Hardware. IEEE, 2003, pp. 199-207.

[13] A. Christensen, R. OǴrady, M. Birattari, and M. Dorigo, "Fault detection in autonomous robots based on fault injection and learning," Autonomous Robots, vol. 24, no. 1, pp. 49-67, 2008.

[14] A. Christensen, R. O'Grady, M. Birattari, and M. M. Dorigo, "Automatic synthesis of fault detection modules for mobile robots." in AHS, 2007, pp. 693-700.

[15] M. Mokhtar, R. Bi, J. Timmis, and A. Tyrrell, "A modified dendritic cell algorithm for on-line error detection in robotic systems," in IEEE Congress on Evolutionary Computation. IEEE, 2009, pp. 2055-2062.

[16] A. Christensen, R. OGrady, and M. Dorigo, "From fireflies to faulttolerant swarms of robots," IEEE Transactions on Evolutionary Computation, vol. 13, no. 4, pp. 754-766, 2009.

[17] B. Jakimovski and E. Maehle, "Artificial immune system based robot anomaly detection engine for fault tolerant robots," in International Conference on Autonomic and Trusted Computing. Springer, 2008, pp. 177-190.

[18] D. Tarapore, P. Lima, J. Carneiro, and A. Christensen, "To err is robotic, to tolerate immunological: fault detection in multirobot systems," Bioinspiration \& biomimetics, vol. 10, no. 1, p. 016014, 2015.

[19] A. Khadidos, R. Crowder, and P. Chappell, "Exogenous fault detection and recovery for swarm robotics," IFAC-PapersOnLine, vol. 48, no. 3, pp. 2405-2410, 2015.

[20] A. Millard, J. Timmis, and A. Winfield, "Run-time detection of faults in autonomous mobile robots based on the comparison of simulated and real robot behaviour," in 2014 IEEE/RSJ International Conference on Intelligent Robots and Systems. IEEE, 2014, pp. 3720-3725.

[21] F. Harrou, L. Fillatre, and I. Nikiforov, "Anomaly detection/detectability for a linear model with a bounded nuisance parameter," Annиal Reviews in Control, vol. 38, no. 1, pp. 32-44, 2014.

[22] V. Venkatasubramanian, R. Rengaswamy, and S. N. Kavuri, "A review of process fault detection and diagnosis: Part ii: Qualitative models and search strategies," Computers \& Chemical Engineering, vol. 27, no. 3, pp. 313-326, 2003.

[23] S. Yin, S. Ding, X. Xie, and H. Luo, "A review on basic data-driven approaches for industrial process monitoring," IEEE Transactions on Industrial Electronics, vol. 61, no. 11, pp. 6418-6428, 2014.

[24] B. Khaldi, F. Harrou, F. Cherif, and Y. Sun, "Monitoring a robot swarm using a data-driven fault detection approach," Robotics and Autonomous Systems, vol. 97, pp. 193-203, 2017.

[25] X. Wu, V. Kumar, J. R. Quinlan, J. Ghosh, Q. Yang, H. Motoda, G. J. McLachlan, A. Ng, B. Liu, S. Y. Philip et al., "Top 10 algorithms in data mining," Knowledge and information systems, vol. 14, no. 1, pp. $1-37,2008$.

[26] D. Cheng, S. Zhang, X. Liu, K. Sun, and M. Zong, "Feature selection by combining subspace learning with sparse representation," Multimedia Systems, vol. 23, no. 3, pp. 285-291, 2017.

[27] Q. P. He and J. Wang, "Fault detection using the k-nearest neighbor rule for semiconductor manufacturing processes," IEEE Transactions on Semiconductor Manufacturing, vol. 20, no. 4, pp. 345-354, 2007.

[28] T. Cheng, F. Harrou, Y. Sun, and T. Leiknes, "Monitoring influent measurements at water resource recovery facility using data-driven soft sensor approach," IEEE Sensors Journal, vol. 19, no. 1, pp. 342-352, 2019.

[29] Z. Zhou, C. Wen, and C. Yang, "Fault isolation based on k-nearest neighbor rule for industrial processes," IEEE Transactions on Industrial Electronics, vol. 63, no. 4, pp. 2578-2586, 2016. 
[30] F. Harrou, Y. Sun, M. Madakyaru, and B. Bouyedou, "An improved multivariate chart using partial least squares with continuous ranked probability score," IEEE Sensors Journal, vol. 18, no. 16, pp. 67156726, 2018.

[31] E. Martin and A. Morris, "Non-parametric confidence bounds for process performance monitoring charts," Journal of Process Control, vol. 6, no. 6, pp. 349-358, 1996.

[32] B. Khaldi and F. Cherif, "Swarm robots circle formation via a virtual viscoelastic control model," 2016 8th International Conference on Modelling, Identification and Control (ICMIC), pp. 725-730, 2016.

[33] C. Pinciroli, V. Trianni, R. O’Grady, G. Pini, A. Brutschy, M. Brambilla, N. Mathews, E. Ferrante, G. Di Caro, F. Ducatelle et al., "Argos: a modular, parallel, multi-engine simulator for multi-robot systems," Swarm intelligence, vol. 6, no. 4, pp. 271-295, 2012.

[34] M. Bonani, V. Longchamp, S. Magnenat, P. Rétornaz, D. Burnier, G. Roulet, F. Vaussard, H. Bleuler, and F. Mondada, "The marxbot, a miniature mobile robot opening new perspectives for the collectiverobotic research," in Intelligent Robots and Systems (IROS), 2010 IEEE/RSJ International Conference on. IEEE, 2010, pp. 4187-4193.

[35] M. Dorigo, D. Floreano, L. M. Gambardella, F. Mondada, S. Nolfi, T. Baaboura, M. Birattari, M. Bonani, M. Brambilla, A. Brutschy et al., "Swarmanoid: a novel concept for the study of heterogeneous robotic swarms," IEEE Robotics \& Automation Magazine, vol. 20, no. 4, pp. 60-71, 2013.

[36] D. M. Powers, "Evaluation: from precision, recall and f-measure to roc, informedness, markedness and correlation," 2011. 